\section{Proportional fair scheduling with reduced feedback}

\section{R. Patachaianand and K. Sandrasegaran}

An opportunistic feedback technique is proposed for proportional fair (PF) scheduling in a downlink multi-user system. This technique requires each mobile station, the scheduling metric of which is higher than a threshold, to send one-bit feedback for PF scheduling. The scheduler determines the optimal modulation and coding scheme for a scheduled user based on channel quality indicator (CQI) update, which is sent on a longer period than the scheduling period. The simulation results indicate that PF scheduling with the proposed update scheme can achieve similar performance as PF scheduling with full CQI feedback. The feedback overhead can be reduced in comparison with typical PF scheduling provided that an optimal CQI update period is chosen.

Introduction: An efficient scheduling algorithm can achieve multi-user diversity [1]. There are two common scheduling algorithms: maximum throughput (MT) scheduling and proportional fair (PF) scheduling. MT scheduling maximises system throughput by allocating all resources to a

ing slot. This algorithm causes unfairness in a situation where mobile stations (MSs) are uniformly distributed over an area because a user located close to the base-station (BS) has a higher chance to be scheduled than those located far away from the BS. On the other hand, the PF algorithm can maintain fairness among all MSs regardless of their location but it has a relatively small sum-capacity compared with MT.

In MT and PF algorithms, all the available resources would be allocated to a user who has the highest scheduling metric in a scheduling time slot. The scheduling metrics for MT and PF are calculated as follows [2, 3]:

$$
\begin{aligned}
& \text { f }
\end{aligned}
$$

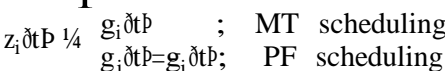

where $\mathrm{g}_{\mathrm{i}} \mathrm{t}_{\mathrm{t}}$ and $\mathrm{g}_{\mathrm{i}} \mathrm{ttp}_{\mathrm{t}}$ denote the instantaneous signal-to-noise ratio (SNR) and average SNR of the ith user at the tth scheduling time slot, respectively. The scheduler in a system employing MT or PF needs to know the scheduling metrics of the users so that the scheduling can be efficiently performed. In a downlink system, the metrics are computed at MSs and delivered to the corresponding BS by means of channel quality indicator (CQI) via a feedback channel. CQI information sent from every MS at every scheduling interval would cause excessive overhead to the system because only one user that has the highest metric will be selected, and CQI from all other users would be discarded.

Several techniques have been proposed to reduce the feedback overhead. For example, in [4], it has been shown that only allowing users whose scheduling metrics are higher than a threshold to send CQI feedback can considerably reduce system feedback overhead, if the optimal threshold is selected. This threshold is determined by the scheduler and broadcast to MSs. The overhead can be further reduced by allowing each user whose scheduling metrics are higher than a threshold to send onebit feedback to the scheduler. It has been shown that MT scheduling based on one-bit feedback can achieve multi-user diversity gain [2,4,5]. In MT scheduling systems, one-bit information is sufficient to perform scheduling because the metric can be mapped to an SNR, which can be directly mapped to a modulation and coding scheme (MCS). PF using one-bit feedback is not feasible because it uses normalised SNR as a scheduling metric and this does not reflect the absolute SNR or supportable MCS. Therefore PF scheduling needs additional information for it to use one-bit feedback. In this Letter, a feedback mechanism for PF scheduling is presented.

Proposed feedback scheme: We propose a CQI update algorithm in which (a) one-bit feedback indicating that the instantaneous normalised SNR of a user is higher than a threshold is sent in every scheduling time slot $\left(\mathrm{T}_{\mathrm{s}}\right)$ and (b) CQI containing several bits indicating a supportable MCS, which is sent on a longer update period $\left(T_{c}\right)$ as shown in Fig. 1. Fig. 1 shows the update sequence of the proposed scheme where $T_{s}$ and $T_{c}$ represent the scheduling period and CQI update period, respectively.

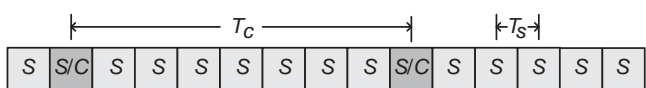

Fig. 1 Update sequence

In the proposed scheme, each MS determines an optimum MCS, taking into account the threshold, and sends the MCS as CQI feedback on a $T_{c}$ basis. The BS then selects a scheduled user in each scheduling slot using one-bit feedback under PF policy and uses the optimal MCS sent in the CQI feedback. Fig. 2 shows the use of one-bit feedback $\left(B_{s}\right)$ and CQI feedback $\left(\mathrm{B}_{\mathrm{c}}\right)$. On every scheduling time slot, one-bit feedback is sent to indicate that the current scheduling metric of a user exceeds a threshold $\left(\mathrm{Z}_{\mathrm{th}}\right)$. $\mathrm{B}_{\mathrm{c}}$ is used to indicate the optimal MCS for each user.

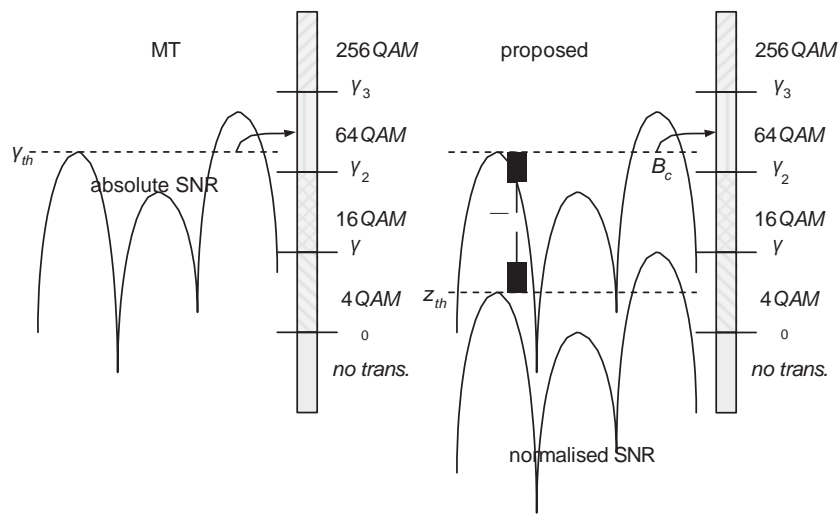

Fig. 2 Use of one-bit feedback and CQI

Scheduling performance: The performance of the proposed technique is evaluated and compared with a typical PF by means of computer simulation. A multi-user downlink packet scheduling for the high data rate (HDR) system with a scheduling slot equal to $1.67 \mathrm{~ms}$ is considered. The instantaneous SNR for each connection is an IID Rayleigh random variable. The users' average SNRs are uniformly distributed $g_{i}$ [ $\left.1 / 2 ; 20\right]$ $\mathrm{dB}$ and assumed to be constant over a user session. Five MCS schemes are used: $\mathbf{M}[\mathbf{~}[0,2,4,6,8]$, where $M$ is the number of bits per symbol (uncoded). M $1 / 40$ represents no transmission mode. Error-free and delayfree feedback channel with perfect channel estimation is assumed throughout this work. It is assumed that the optimum feedback threshold is selected so that the achievable sum-capacity can be realised.

Fig. 3 shows the sum-rate capacity of the proposed one-bit feedback technique with optimum threshold. We can see that the proposed technique has the same capacity growth rate and achieves similar sum-rate capacity as the PF with full CQI feedback. However, the proposed technique achieves this performance by minimising the required feedback. The analysis of the reduced feedback load is discussed in the following section.

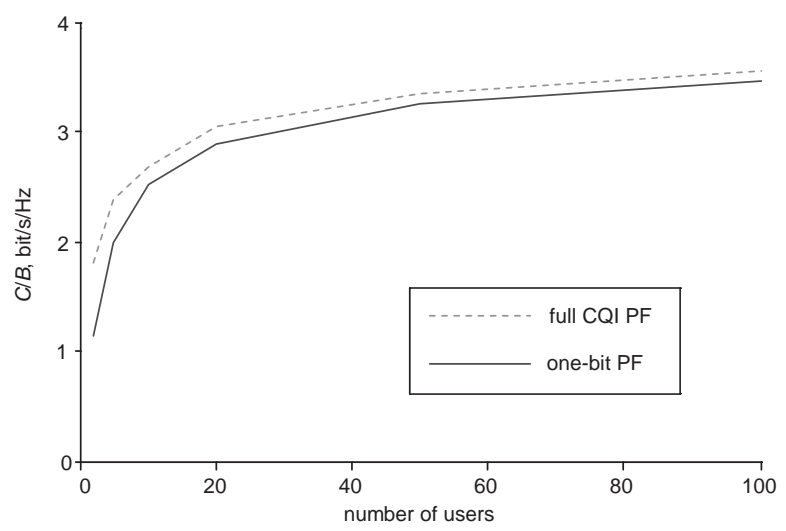

Fig. 3 Performance comparison

CQI updating period: All users are required to report CQI on a relatively long update interval $\left(T_{c}\right)$ compared with scheduling time slot $\left(T_{\mathrm{s}}\right)$. It is critical to determine an optimum CQI update period that does not introduce excessive feedback overhead. The feedback overhead 
of the proposed technique is compared with the overhead of opportunistic feedback where users whose scheduling metrics are greater than a threshold send CQI feedback to the BS (hereafter OCF: opportunistic CQI feedback). To simplify our analysis, it is assumed that the number of users who send feedback could be limited to a certain value. Note that given the number of feedback users are the same for both cases, OCF is more sensitive to an increased number of feedback users since more bits are sent per an additional feedback user. The overhead comparison of the two techniques is based on the ratio of the number of feedback bits required for each scheme, RFB, as given below:

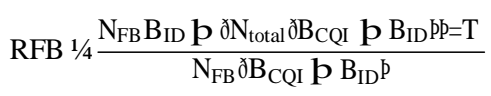

or

$$
\mathrm{RFB}^{1 / 4} \frac{1}{1 \mathbf{p}^{\mathrm{B}} \mathrm{CQI}=\mathrm{B}_{\mathrm{ID}}} \mathbf{P} \frac{1}{\mathrm{TN}_{\mathrm{FB}}=\mathrm{N}_{\text {total }}}
$$

where $\mathrm{N}_{\mathrm{FB}}$ represents the number of users whose scheduling metrics are above the threshold. $\mathrm{N}_{\text {total }}$ is the total number of active users in the system. $\mathrm{B}_{\mathrm{ID}}$ and $\mathrm{B}_{\mathrm{CQI}}$ are the number of bits representing user identity and CQI, respectively. Tis the ratio of the CQI update period to the scheduling period $\left(T_{c} / T_{s}\right)$. In the comparative analysis, the following parameters are assumed: $\mathrm{B}_{\mathrm{CQI}} 1 / 45$, and $\mathrm{B}_{\mathrm{ID}} \frac{1 / 4}{40}$ for maximum 100 active users. Scheduling interval, $T_{\mathrm{s}}$, is $1.67 \mathrm{~ms} . \mathrm{N}_{\mathrm{FB}}$ is assumed to be a constant at five users. Note that this assumption is advantageous for OCF since more bits are sent per one feedback user.

Fig. 4 shows the ratio between the feedback overhead of the proposed technique and opportunistic CQI feedback technique (as given in (3)) against $T_{c} / T_{s}$. This ratio reduces when the $T_{c} / T_{s}$ value increases because less frequent CQI feedback is sent. The intersection between the solid line and the dash-dotted line in Fig. 4 is the position at which the feedback load of the proposed technique equals that of the OCF, which is corresponding to one CQI update period per 60 scheduling slots $\mathrm{T}_{\mathrm{s}}$. It can be seen that CQI needs to be updated once per 150 scheduling slots on average to achieve a $20 \%$ overhead reduction (80\% load ratio on y-axis in Fig. 4) in comparison to OCF. This update period corresponds to $0.25 \mathrm{~s}$ given that the scheduling period is $1.67 \mathrm{~ms}$. This update period is sufficient to cope with long-term fading such as shadowing and path loss.

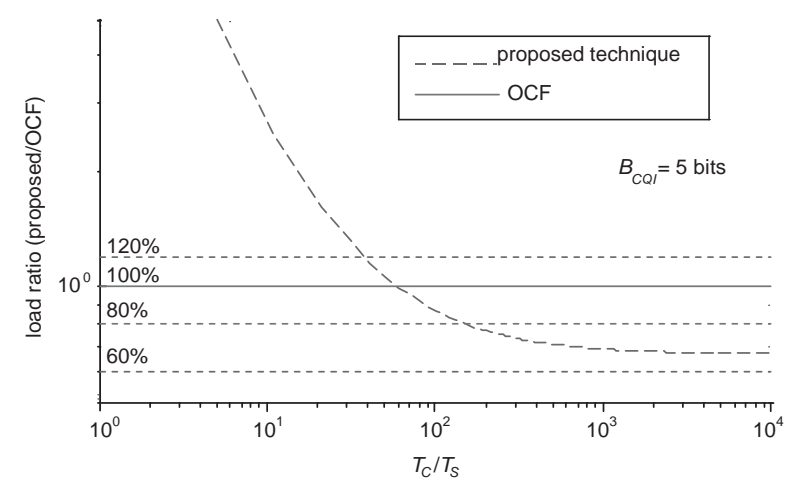

Fig. 4 Feedback overhead comparison
Conclusions: An opportunistic feedback technique is presented for PF scheduling. The technique requires each MS, the scheduling metric of which is higher than a threshold, to send one-bit feedback for PF scheduling. The BS determines the optimal MCS for a scheduled user by CQI update, which is based on a relatively long period compared with the scheduling slot. The simulation results indicate that PF scheduling with the proposed update scheme can achieve similar performance as PF scheduling with full CQI feedback. The feedback overhead can be reduced in comparison with OCF if an optimal CQI update period is chosen.

\section{doi: 10.1049/el.2009.3522}

R. Patachaianand and K. Sandrasegaran (Faculty of Engineering and Information Technology, University of Technology Sydney, 1 Broadway, Ultimo, Sydney, Australia)

E-mail: rpatacha@eng.uts.edu.au

\section{References}

1 Viswanath, P., Tse, D.N.C., and Laroia, R.: 'Opportunistic beamforming using dumb antennas', IEEE Trans. Inf. Theory, 2002, 48, (6), pp. 1277-1294

2 Choi, J.-G., and Bahk, S.: 'Cell-throughput analysis of the proportional fair scheduler in the single-cell environment', IEEE Trans. Veh. Technol., 2007, 56, (2), pp. 766-778

3 Yang, L., Kang, M., and Alouini, M.-S.: 'On the capacity-fairness tradeoff in multiuser diversity systems', IEEE Trans. Veh. Technol., 2007, 56, (4), pp. 1901-1907

4 Gesbert, D., and Alouini, M.-S.: 'How much feedback is multi-user diversity really worth?'. Proc. IEEE Int. Conf. on Communications (ICC '04), Paris, France, June 2004, pp. 234-238

5 Sanayei, S., and Nosratinia, A.: 'Opportunistic downlink transmission with limited feedback', IEEE Trans. Inf. Theory, 2007, 53, (11), pp. 4363-4371 